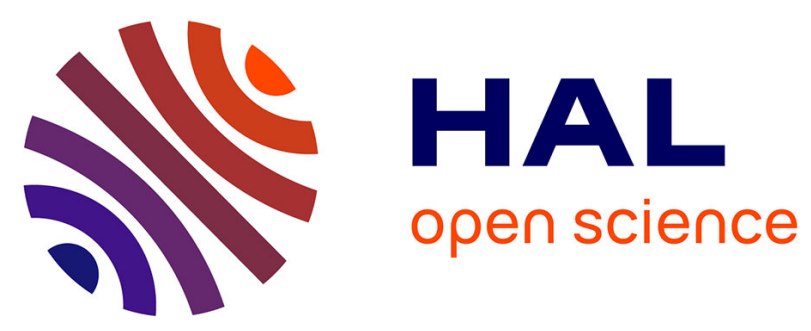

\title{
System identification of the intracellular photoreaction process induced by photodynamic therapy
}

Simona Dobre, Thierry Bastogne, Muriel Barberi-Heyob, Denise Bechet, Jacques Didelon, Alain Richard

\section{To cite this version:}

Simona Dobre, Thierry Bastogne, Muriel Barberi-Heyob, Denise Bechet, Jacques Didelon, et al.. System identification of the intracellular photoreaction process induced by photodynamic therapy. 16th Mediterranean Conference on Control and Automation, MED'08, Jun 2008, Ajaccio, France. pp.1729-1734，10.1109/MED.2008.4602185 . hal-00322893

\section{HAL Id: hal-00322893 https://hal.science/hal-00322893}

Submitted on 19 Sep 2008

HAL is a multi-disciplinary open access archive for the deposit and dissemination of scientific research documents, whether they are published or not. The documents may come from teaching and research institutions in France or abroad, or from public or private research centers.
L'archive ouverte pluridisciplinaire HAL, est destinée au dépôt et à la diffusion de documents scientifiques de niveau recherche, publiés ou non, émanant des établissements d'enseignement et de recherche français ou étrangers, des laboratoires publics ou privés. 


\title{
System identification of the intracellular photoreaction process induced by photodynamic therapy
}

\author{
Simona Dobre*, Thierry Bastogne*, Muriel Barberi-Heyob ${ }^{\dagger}$, Denise Bechet ${ }^{\dagger}$, Jacques Didelon ${ }^{\ddagger}$, Alain Richard* \\ *Centre de Recherche en Automatique de Nancy (CRAN), Nancy - Université, CNRS UMR 7039, \\ BP 239, F-54506 Vandœuvre-lès-Nancy Cedex, France, \\ Simona.Dobre; Thierry. Bastogne; Alain.Richardecran.uhp-nancy. fr \\ †Centre de Recherche en Automatique de Nancy (CRAN), Nancy - Université, CNRS UMR 7039, \\ Centre Alexis Vautrin, 54511 Vandœuvre-lès-Nancy Cedex, France, \\ m.barberi@nancy.fnclcc.fr \\ ‡Centre Alexis Vautrin, Laboratoire IMAC, "Mesures photophysiques et instrumentation", \\ 54500 Vandœuvre-lès-Nancy Cedex, France
}

\begin{abstract}
Photodynamic therapy (PDT) is an alternative treatment for cancer that involves the administration of a photosensitizing agent, which is activated by light at a specific wavelength. This illumination causes a sequence of photoreactions, which - in the presence of molecular oxygen - is supposed to be responsible for the death of the tumor cells. The PDT efficiency stems from the optimal interaction between these three factors (light, drug and oxygen). In this paper, a new approach is proposed to estimate photophysical parameters which characterize the ability of a photosensitizing drug to produce singlet oxygen. This approach is based on system identification techniques. This model-based method would allow biologists to estimate all the photophysical parameters from spectro-fluorescence data generated by only one experiment. Secondly, contrary to usual techniques which are restricted to in vitro studies, this approach can be directly applied to in vivo data.
\end{abstract}

\section{INTRODUCTION}

Photodynamic therapy (PDT) [1], [2], [12] is a treatment of displastic tissues such as cancers. Mainly, it involves the selective uptake and retention of the photosensitizing drug (photosensitizer, PS) in the tumor, followed by its illumination with light of appropriate wavelength. The PS activation is thought to produce, after multiple intermediate reactions, singlet oxygen at high doses (in the presence of molecular oxygen) and thereby to initiate apoptotic and necrotic death of tumor.

Currently, the efficiency of the photodynamic phase (production of singlet oxygen), as well as the comparison of different PS, are based on three photophysical characteristics:

- the absorption coefficient, $\sigma_{S}$, of the PS in the spectral region of the excitation light. $\sigma_{S}$ characterizes the PSlight interaction;

- the quantum yield of the PS triplet state, $\Phi_{T}$ and the triplet state lifetime, $\tau_{T}$;

- the quantum yield of singlet oxygen production, $\Phi_{\Delta}$ which characterizes the PS-oxygen interaction.

These photophysical coefficients were till now separately determined from in vitro dedicated experiments ([10], [9]), but they cannot always predict the in vivo cytotoxic action.
In this paper, a model-based method, based on system identification techniques [14], is proposed as an alternative and complementary approach for the estimation of PDT photophysical characteristics. Such an approach could provide two main benefits: (1) the estimation of the photophysical coefficients directly from in vivo experiments and (2) a significant cut of the experimental cost by decreasing the number of trials. So far, the estimation of the photophysical coefficients by system identification techniques has never been assessed in practice.

Five main difficulties can be addressed for the application of such a solution to in vivo spectro-fluorescence data:

- the nonlinearity structure of the photopohysical model;

- the large range of possible values (up to four decades) for the unknown parameters;

- some initial state variables are unknown;

- the lack of information (only one measured variable over six state variables);

- the limited degrees-of-freedom for the choice of the laser light stimulus (input variable), see paragraph II$\mathrm{C}$ for more information about the experimental setup.

A dynamic model of the photoreaction phase, the relationships between the photophysical characteristics and the model parameters, and the experimental setup are introduced in section II. Section III is devoted to the experimetal modeling methodology while section IV deals with the selection of practically identifiable parameters. The estimation of the model parameters, the model falsification tests and the determination of the parameter uncertainty are presented in sections V, VI and VII respectively. A discussion of the results is provided before to conclude.

\section{Problem Statement}

As previously mentioned in the introduction, the interaction between the light (at a specific wavelength) and the PS already incorporated in the tumor, determines a sequence of photoreactions which are presumed to have as result the 
death of the tumor cells (through the production of singlet excited oxygen - a very reactive specie).

\section{A. Model Description}

Based on the kinetics equations describing the type-II reactions specific to PDT [1], [2], [7], a nonlinear state-space model of the intracellular photoreaction process may be introduced. This model is composed of six state variables and eleven unknown photophysical parameters, and is described as follows,

$$
\left\{\begin{aligned}
\frac{d\left[S_{0}\right]}{d t}= & u_{P}+k_{f}\left[S_{1}\right]-k_{P b}\left[S_{0}\right]\left[{ }^{1} O_{2}\right]+ \\
& +k_{p}\left[T_{1}\right]+k_{T}\left[T_{1}\right]\left[{ }^{3} O_{2}\right]-k_{A} u_{L}\left[S_{0}\right] \\
\frac{d\left[S_{1}\right]}{d t}= & k_{A} u_{L}\left[S_{0}\right]-\left(k_{f}+k_{I S C}\right)\left[S_{1}\right]- \\
& -k_{S M}\left[S_{1}\right][M] \\
\frac{d\left[T_{1}\right]}{d t}= & k_{I S C}\left[S_{1}\right]-k_{p}\left[T_{1}\right]-k_{T}\left[T_{1}\right]\left[{ }^{3} O_{2}\right]- \\
& -k_{T M}\left[T_{1}\right][M] \\
\frac{d\left[{ }^{3} O_{2}\right]}{d t}= & u_{O}-k_{T}\left[T_{1}\right]\left[{ }^{3} O_{2}\right]+k_{l}\left[{ }^{1} O_{2}\right] \\
\frac{d\left[{ }^{1} O_{2}\right]}{d t}= & k_{T}\left[T_{1}\right]\left[{ }^{3} O_{2}\right]-k_{l}\left[{ }^{1} O_{2}\right]- \\
& -k_{O x}[M]\left[{ }^{1} O_{2}\right]-k_{P b}\left[S_{0}\right]\left[{ }^{1} O_{2}\right] \\
\frac{d[M]}{d t}= & -k_{O x}[M]\left[{ }^{1} O_{2}\right]-k_{S M}\left[S_{1}\right][M]- \\
& -k_{T M}\left[T_{1}\right][M] \\
y\left(t_{i}\right)= & \gamma\left[S_{0}\right]\left(t_{i}\right)+v\left(t_{i}\right) \\
v\left(t_{i}\right) \sim & \mathscr{N}\left(0, s^{2}\right)
\end{aligned}\right.
$$

where $x(t, p)=\left[\left[S_{0}\right],\left[S_{1}\right],\left[T_{1}\right],\left[{ }^{3} O_{2}\right],\left[{ }^{1} O_{2}\right],[M]\right]^{T} \in \mathbb{R}^{6}$ are the state variables; the light input $u_{L}(t)$ corresponds the irradiance signal. The incorporation of PS molecules into cancer cells is a slow dynamic process, comparatively with the photoreactions. Accordingly, the PS uptake rate, $u_{P}$, is assumed to be null during the experiment, i.e. during a relatively short period of time, $<1 h$. The oxygen input $u_{O}(t)$ is not manipulated and measured. In this application, $u_{O}(t)$ is regarded as a disturbance variable. The output noise $v\left(t_{i}\right)$ is assumed to be a realization sequence of independent and identically distributed gaussian variables at the measurement times $\left\{t_{i}\right\} . p=\left[k_{I S C}, k_{P b}, k_{l}, k_{f}, k_{p}, k_{A}, k_{o x}, k_{T M}, k_{S M}, k_{T}, \gamma\right]^{T} \in$ $\mathbb{R}^{11}$ are the unknown parameters of the model, with $k_{f}=$ $k_{F}+k_{I C}, k_{p}=k_{P}+k_{T S}$ and $k_{l}=k_{l}^{n r}+k_{l}^{r}$. Tab. I contains the model variables description whereas Tab. II presents all the parameters with their initial guess (see step 2 of the experimental modeling procedure) and the ones of the state variables.

\section{B. Photophysical Parameters}

The relationships between the model parameters and the photophysical coefficients are:

$$
\begin{gathered}
k_{A}=\frac{\sigma_{S}}{h v_{A}} \Rightarrow \sigma_{S}=h \cdot v_{A} \cdot k_{A} \\
\Phi_{T}=\frac{k_{I S C}}{k_{I S C}+k_{f}} ; \tau_{T}=\frac{1}{k_{I S C}+k_{f}} \\
\Phi_{\Delta}=\Phi_{T} \cdot \phi_{e t}=\frac{k_{I S C}}{k_{I S C}+k_{f}} \cdot \frac{k_{T}\left[{ }^{3} O_{2}\right]}{k_{T}\left[{ }^{3} O_{2}\right]+k_{p}}
\end{gathered}
$$

where $h=6.026 \cdot 10^{-34} \mathrm{~J} \cdot s$ is Planck's constant, $v_{A}$ is the frequency of the incident light, $\phi_{e t}$ is the efficiency of the energy transfer to molecular oxygen. Note that for a given concentration of molecular oxygen $\left[{ }^{3} \mathrm{O}_{2}\right]$, all the
TABLE I

MODEL VARIABLES

\begin{tabular}{|c|c|c|}
\hline Symbol & Definition & Units \\
\hline \hline$\left[S_{0}\right]$ & Photosensitizer ground state & $\mathrm{mM}$ \\
\hline$\left[S_{1}\right]$ & Photosensitizer singlet excited state & $\mathrm{mM}$ \\
\hline$\left[T_{1}\right]$ & Photosensitizer triplet excited state & $\mathrm{mM}$ \\
\hline$\left.{ }^{3} O_{2}\right]$ & Triplet ground - state oxygen & $\mathrm{mM}$ \\
\hline${ }^{1} O_{2}$ & Singlet excited - state oxygen & $\mathrm{mM}$ \\
\hline$[M]$ & Cellular targets & $\mathrm{mM}$ \\
\hline$u_{P}$ & Uptake rate of photosensitising molecules & $\mathrm{mM} \cdot \mathrm{s}^{-1}$ \\
\hline$u_{L}$ & Irradiance signal & $\mathrm{W} \cdot \mathrm{cm}^{-2}$ \\
\hline$u_{O}$ & Uptake rate of oxygen molecules & $\mathrm{mM} \cdot \mathrm{s}^{-1}$ \\
\hline$y$ & Fluorescence Intensity & $\mathrm{a} \cdot \mathrm{u}$ \\
\hline
\end{tabular}

\begin{tabular}{|c|c|c|}
\hline Symbol & Definition & Values \\
\hline$\overline{k_{A}}$ & PS absorption rate at $u_{L}=76 \mathrm{~mW} / \mathrm{cm}^{2}$ & 19 \\
\hline$k_{P b}$ & Photobleaching rate & $\begin{array}{l}1.2 \cdot 10^{8} \\
m M^{-1} s^{-1}\end{array}$ \\
\hline$k_{T}$ & $\begin{array}{l}\text { Biomolecular rate constant for } \\
\text { the reaction of }{ }^{3} \mathrm{O}_{2} \text { with } T_{1}\end{array}$ & $\begin{array}{l}10^{5} \\
m M^{-1} s^{-1}\end{array}$ \\
\hline$k_{f}$ & Rate constant for the reaction $S_{1} \rightarrow S_{0}$ & $2 \cdot 10^{7} s^{-1}$ \\
\hline$k_{p}$ & Rate constant for the reaction $T_{1} \rightarrow S_{0}$ & $1250 s^{-1}$ \\
\hline$k_{I S C}$ & Rate constant for the reaction $S_{1} \rightarrow T_{1}$ & $8 \cdot 10^{7} s^{-1}$ \\
\hline$k_{l}$ & Rate constant for the reaction ${ }^{1} \mathrm{O}_{2} \rightarrow{ }^{3} \mathrm{O}_{2}$ & $10^{6} s^{-1}$ \\
\hline$k_{o x}$ & $\begin{array}{l}\text { Bimolecular rate constant for } \\
\text { the reactions }{ }^{1} \mathrm{O}_{2} \text { with } M\end{array}$ & $\begin{array}{l}2.6 \cdot 10^{9} \\
m M^{-1} s^{-1}\end{array}$ \\
\hline$k_{S M}$ & $\begin{array}{l}\text { Bimolecular rate constant for } \\
\text { the reaction of } S_{1} \text { with } M\end{array}$ & $\begin{array}{l}10^{-2} \\
m M^{-1} s^{-1}\end{array}$ \\
\hline$k_{T M}$ & $\begin{array}{l}\text { Bimolecular rate constant for } \\
\text { the reaction of } T_{1} \text { with } M\end{array}$ & $1 m M^{-1} s^{-1}$ \\
\hline$\gamma$ & measurement coefficient & 43.15 a.u. \\
\hline$\left[S_{0}\right]_{0}$ & Initial condition for $\left[S_{0}\right]$ & $8.5 \cdot 10^{-3} \mathrm{mM}$ \\
\hline$\left[S_{1}\right]_{0}$ & Initial condition for $\left[S_{1}\right]$ & $0 \mathrm{mM}$ \\
\hline$\left[T_{1}\right]_{0}$ & Initial condition for $\left[T_{1}\right]$ & $0 m M$ \\
\hline$\left.{ }^{3} 0_{2}\right]_{0}$ & Initial condition for $\left[{ }^{3} \mathrm{O}_{2}\right]$ & $83 \cdot 10^{-3} \mathrm{mM}$ \\
\hline $\begin{array}{l}\left.{ }^{\mathrm{T}} \mathrm{O}_{2}\right]_{0} \\
\end{array}$ & Initial condition for $\left[{ }^{1} \mathrm{O}_{2}\right]$ & $0 \mathrm{mM}$ \\
\hline$[M]_{0}$ & Initial condition for $[M]$ & $830 \cdot 10^{-3} \mathrm{mM}$ \\
\hline
\end{tabular}

TABLE II

NOMinal Parameter VALUeS AND INITIAL CONDITIONS For THE PDT-PHOTOREACTION MODEL

photophysical coefficients can be deduced from $p$, the vector of the model parameters.

\section{Problem statement}

As previously pointed out, the in vivo determination of $\sigma_{S}$, $\Phi_{T}, \tau_{T}$ and $\Phi_{\Delta}$ can be expressed as a problem of identifiability and estimation of $p$ from the in vivo measurement of $u_{L}$ and $y$.

\section{Experimental Setup}

Fig. 1 shows the in vivo experimental setup. Female athymic Foxn1 nude mice were used for this study (Harlan, Gannat, France). The mice were used for tumor implantation when they were $7-9$ weeks old $(20-25 g)$. The model of human malignant glioma was obtained using U87 cells, as described in [22]. Tumors were treated when they reached $5 \pm 1 \mathrm{~mm}$ ( $15 \pm 5$ days after tumor grafting). The photosensitizing molecule is TPC-Ahx-ATWLPPR [23] and 


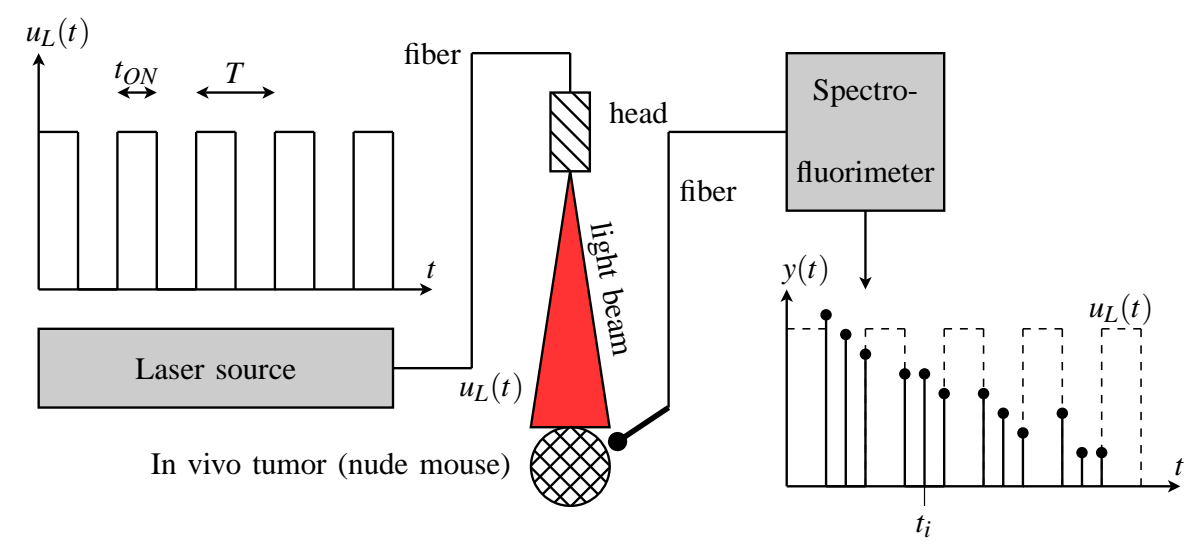

Fig. 1. in vitro experimental set up

the administrated dose, $u_{P}=1.75 \mu \mathrm{mol} \cdot \mathrm{kg}^{-1}$, was injected to the mice via the tail vein. The mice were kept in the dark for $4 h$, and anesthetized. Irradiation of tumors was carried out at $\lambda=652 \mathrm{~nm}$, using a dye laser, pumped with an argon laser. Light was delivered through an optical fiber and illuminated the tumor surface over a $1 \mathrm{~cm}$ diameter beam spot. The incident fluence rate on the tumors was previously evaluated using an optical power meter. The fluence rate (irradiance) $u_{L}(t)$ is a square wave signal. Its magnitude reaches $95 \mathrm{~mW} \cdot \mathrm{cm}^{-2}$ and its period is equal to $T=1 \mathrm{mn}$ with a $50 \%$ duty cycle. The fluorescence intensity $y(t)$ is collected by a spectrofluorimeter only during the dark phases (laser off) and with a sampling period of time equal to $T_{s}=7 \mathrm{~s}$, i.e. three points for each dark half cycle (technical limit of the data acquisition system). The total duration of the experiment is limited to about $42 \mathrm{~min}$.

\section{Experimental Modeling Methodology}

The experimental modeling procedure used in this application study has been presented in [8]. The latter approach is composed of six steps,

1) Defining the model structure and assessing its theoretical (or structural) identifiability [14] deals with the possibility to give an unique value to each parameter of a mathematical model structure. The uniqueness of this solution is assessed in an idealized or theoretical framework where the process and the model have identical structures, the data is noise-free, and where the input signals and the measurement times can be chosen at will. In this application, the global structural identifiability of model (1) was checked by the similarity transformation method.

2) A prior analysis, often based on a literature analysis, is carried out to provide the initial guess of $p$. In order to improve the numerical properties of the Fisher Information Matrix (see section IV), a rescaling of the parameters is done: $p_{i}=p_{i}^{0} \cdot \Theta_{i}$, with $p^{0}$ initial guess for the parameters, $\Theta$ the normalized parameters to be estimated and $i=1, \cdots, 11$.

3) The third step is devoted to the implementation of the model into a simulation environment. In this case, the simulation model is represented by a block-diagram implemented into Simulink ${ }^{\complement} 1$.

4) Structural identifiability is a qualitative concept. When the model is proved structurally identifiable, an admissible experiment has to be selected to collect the estimation data. The design of optimal experiments may be regarded as maximizing a measure of identifiability, thereby transforming the intitial qualitative problem into a quantitative one, also called practical identifiability [15], [19]. In a fourth step, a practical identifiability analysis is locally carried out through the evaluation of the output sensitivity functions and the corresponding Fisher Information Matrix [20], [8]. The output sensitivity functions are computed by the software Diffedge ${ }^{(\mathcal{C}}$ which enables the sensitivity analysis of block diagrams by computer algebra [18].

5) In the next step, the practically identifiable parameters $\bar{\Theta}$ are selected according to numerical properties of an empirical Fisher information Matrix.

6) The sixth step deals with the parameter estimation. The estimation criterion is defined as follows:

$$
J(\Theta)=\sum_{j=1}^{N} e\left(t_{j}, \Theta\right)^{T} \cdot e\left(t_{j}, \Theta\right)
$$

where $\bar{\Theta}$ is a subvector of $\Theta$ containing the selected parameters to be estimated and $e(t, \Theta)$ is the output error defined by:

$$
e(t, \Theta)=y_{S}(t)-y(t, \Theta) \sim \mathscr{N}\left(0, \sigma^{2}\right)
$$

with $y_{S}(t)$ the output system measurement. $\left\{e\left(t_{j}, \Theta\right) \mid j=1, \cdots, N\right\}$ is assumed to be a realization sequence of independent and identically distributed gaussian variables. $\left\{t_{j} \mid j=1, \cdots, N\right\}$ is the sequence of the measurement time instants.

7) The last step consists in estimating the empirical probability density functions of the identifiable model parameters in order to characterize their uncertainty.

\footnotetext{
${ }^{1}$ Simulink builds upon Matlab ${ }^{\complement}$, The MathWorks, Inc
} 


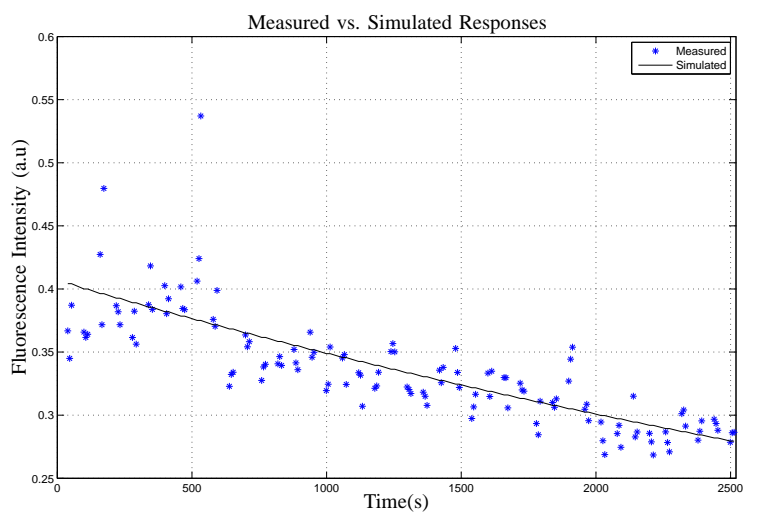

Fig. 2. Measured vs Estimated Responses

\section{SELECTION OF IDENTIFIABLE PARAMETERS}

The selection of the practically identifiable parameters relies on an empirical Fisher Information Matrix defined by

$$
F I M \propto \sum_{j=1}^{N}\left(\frac{\partial y\left(t_{j}, \Theta\right)}{\partial \Theta}\right)^{T}\left(\frac{\partial y\left(t_{j}, \Theta\right)}{\partial \Theta}\right)
$$

The number of practically identifiable parameters is given by the numerical rank of FIM. Given $u_{L}$ and the measurement time instants defined in section II-D, the numerical rank of FIM was equal to 11 , meaning that all the parameters are practically identifiable.

\section{PARAMETER ESTIMATION}

The parameter estimation step was based on a least squares method applied to the output error. The minimization of the estimation criterion (3) with model (1) as a constraint was solved using an Active-Set algorithm implemented in Matlab. Parameter estimates are given in Tab. (III). Figure 2 shows the measured values vs the estimated responses.

\section{MODEL FALSIFICATION}

The model falsification test used herein consists in checking the modeling assumption, stated in section II-A, about the gaussian distribution of the output residuals. Two statistical tests were implemented. The first one is a quantilie-quantile plot and the second test is based on the Pearsons chi-square test.

Let us firstly formulate the null hypothesis $\left(H_{0}\right)$ and the alternative hypothesis $\left(H_{1}\right)$ such that:

$$
\begin{cases}H_{0}: & \mathbf{e} \sim \mathscr{N}\left(0, \sigma^{2}\right) \\ H_{1}: & \mathbf{e} \nsim \mathscr{N}\left(0, \sigma^{2}\right)\end{cases}
$$

where e denotes a random variable of which an observed sequence is $\left\{e\left(t_{i}\right) \mid i=1, \cdots, N\right\}$.

\section{A. $Q-Q$ test}

A Quantile-Quantile plot is a graphical method for diagnosing differences between the probability distribution of a statistical population from which a random sample has been taken and a comparison distribution. Herein, The Q-Q test

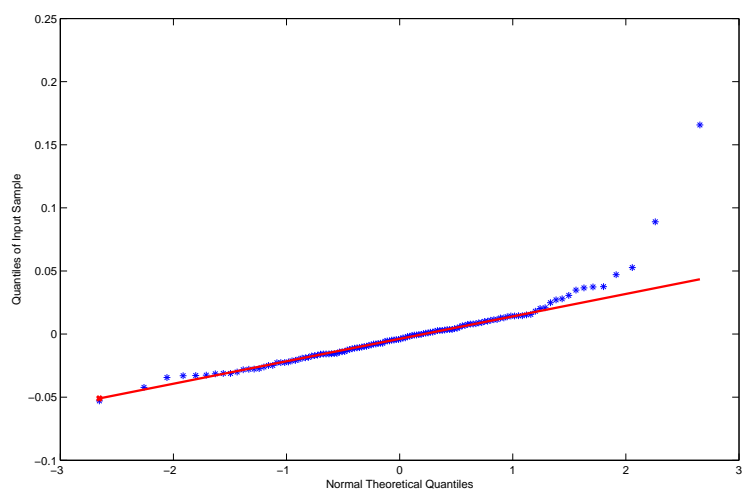

Fig. 3. Q-Q plot of output residuals

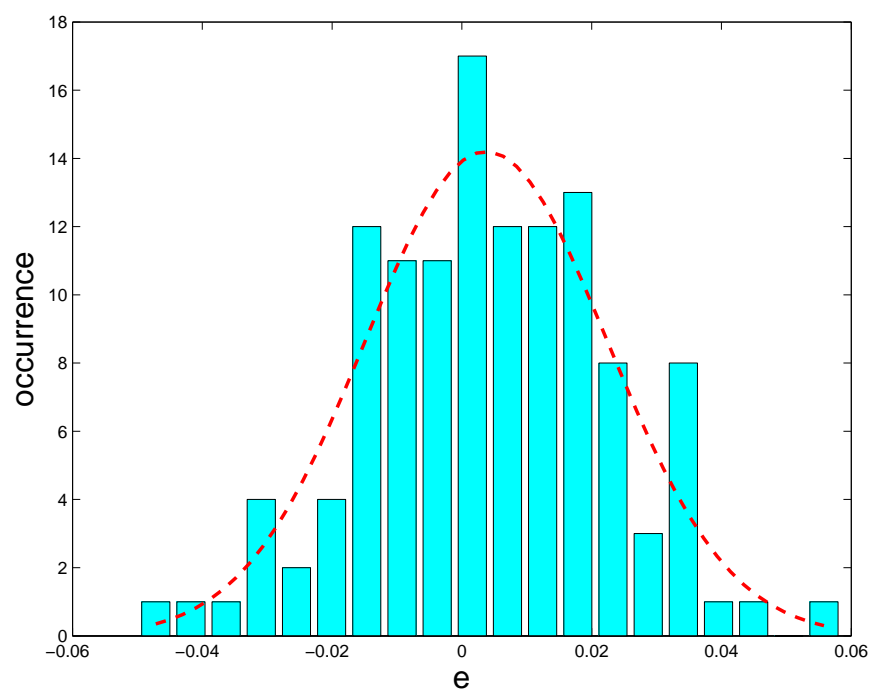

Fig. 4. Histogram of output residuals

is used for assessing whether or not the output residuals are approximately normal distributed.

The data are plotted against a theoretical normal distribution in such a way that the points should form an approximate straight line. Departures from this straight line indicate departures from normality. The results are represented in fig. 3. The normal probability plot shows a reasonably linear pattern in the center of the data, therefore the null hypothesis $H_{0}$ is sustained.

\section{B. Pearsons chi-square test}

As shown in Fig. 4, the sequence of output residuals is discretized into 60 bins. The number of degrees of freedom is $r=18$. The test statistic computed from the observed frequencies (histogram in Fig.4) $\chi^{2}=30.94$ is lower than the critical value $\chi_{\alpha, r}^{2}=32.34$. Accordingly, the null hypothesis cannot be rejected for $\alpha=0.02$. In other words, the model based on the estimated parameters is not falsified.

\section{CRITICAL ANALYSIS OF THE RESUlts}

A possible problem with the proposed approach is that the numerical search may terminate far from the true minimum 
TABLE III

PDT(PHASE 2) ESTIMATION RESULTS

\begin{tabular}{|l|c|c|c|c|c|c|c|c|c|c|c|}
\hline Parameter $p_{i}=p_{i}^{0} \cdot \Theta_{i}$ & $k_{I S C}$ & $k_{P b}$ & $k_{l}$ & $k_{f}$ & $k_{p}$ & $k_{A}$ & $k_{O x}$ & $k_{T M}$ & $k_{S M}$ & $k_{T}$ & $\gamma$ \\
\hline Estim. value $\hat{p}_{i}=p_{i}^{0} \cdot \hat{\Theta}_{i}$ & $7.55 \cdot 10^{7}$ & $1.1 \cdot 10^{8}$ & $9.68 \cdot 10^{5}$ & $2.21 \cdot 10^{7}$ & $1.2 \cdot 10^{3}$ & 13.7 & $2.53 \cdot 10^{9}$ & 0.57 & 0.05 & $4.65 \cdot 10^{4}$ & 46.82 \\
\hline
\end{tabular}

TABLE IV

UNCERTAINTY ANALYSIS RESULTS

\begin{tabular}{|c|c|l|}
\hline $\begin{array}{c}\text { Parameter } \\
\Theta_{i}\end{array}$ & $\begin{array}{c}95 \% \text { confidence interval } \\
{\left[q_{0.025}\left(\Theta_{i}\right), q_{0.975}\left(\Theta_{i}\right)\right]}\end{array}$ & $\begin{array}{l}\text { Parameter } \\
p_{i}\end{array}$ \\
\hline$\Theta_{11}$ & {$[1.0541,1.1156]$} & $p_{11}=\gamma^{0} \cdot \Theta_{11}$ \\
\hline$\Theta_{8}$ & {$[0.5578,0.7212]$} & $p_{8}=k_{T M}^{0} \cdot \Theta_{8}$ \\
\hline$\Theta_{6}$ & {$[0.6046,0.8776]$} & $p_{6}=k_{A}^{0} \cdot \Theta_{6}$ \\
\hline$\Theta_{1}$ & {$[0.7661,1.2636]$} & $p_{1}=k_{I S C}^{0} \cdot \Theta_{1}$ \\
\hline$\Theta_{5}$ & {$[0.8517,1.3626]$} & $p_{5}=k_{p}^{0} \cdot \Theta_{5}$ \\
\hline$\Theta_{7}$ & {$[0.8227,1.3471]$} & $p_{7}=k_{o x}^{0} \cdot \Theta_{7}$ \\
\hline$\Theta_{4}$ & {$[0.7653,1.3682]$} & $p_{4}=k_{f}^{0} \cdot \Theta_{4}$ \\
\hline$\Theta_{2}$ & {$[0.7762,1.4060]$} & $p_{2}=k_{P b}^{0} \cdot \Theta_{2}$ \\
\hline$\Theta_{3}$ & {$[0.7806,1.4539]$} & $p_{3}=k_{l}^{0} \cdot \Theta_{3}$ \\
\hline$\Theta_{10}$ & {$[0.7856,1.4716]$} & $p_{10}=k_{T}^{0} \cdot \Theta_{10}$ \\
\hline$\Theta_{9}$ & {$[4.6648,5.4799]$} & $p_{9}=k_{S M}^{0} \cdot \Theta_{9}$ \\
\hline
\end{tabular}

for several reasons, such as inadequate choice of the optimization algorithm coefficients or initial parameter guess, or trapping in a local minima.

\section{UNCERTAINTY ANALYSIS}

Because of the low signal-to-noise ratio, determining the optimal values of the parameters $\Theta$ with respect to the chosen criterion [14], [6], [21] is not enough. Herein, a MonteCarlo approach was carried out to determine the empirical probability density functions of the parameter estimates. The implemented procedure is the following:

- Based on the experimental data, compute a parameter estimate $\hat{\Theta}$;

- Check the validity of the hypothesis on the noise $e$ (see section VI);

- Generate vectors of fictitious data by running the model with parameters $\hat{\Theta}$ for $N_{\text {sim }}=1000$ realizations of i.i.d. $\mathscr{N}\left(0, \hat{\sigma}^{2}\right)$ variables $\hat{v}\left(t_{i}\right)$ with

$$
\hat{\sigma}^{2}=\frac{1}{N-1} \sum_{i=1}^{N} e\left(t_{i}\right)^{2}
$$

- Estimate $N_{\text {sim }}$ series of estimated parameters $\tilde{\Theta}$ based on each of these vectors of fictitious data $\tilde{y}$ and using the same method as for the experimental data;

- Given the $N_{\text {sim }}$ estimates of $\Theta_{i}$, compute the 25th and 975th empirical 1000-quantiles (permillages), noted $q_{0.025}\left(\Theta_{i}\right)$ and $q_{0.975}\left(\Theta_{i}\right)$. Then, the empirical $95 \%$ confidence interval for $\Theta_{i}$ is given by $\left[q_{0.025}\left(\Theta_{i}\right) ; q_{0.975}\left(\Theta_{i}\right)\right]$.

The empirical $95 \%$ confidence interval for all the parameters estimates are given in Tab. IV. Fig. 5 illustrates all the histograms obtained with the Monte-Carlo method.

\section{CONCLUSION}

In this study, the estimation of the photophysical parameters included in photoreactions induced by PDT is examined. This objective has never been reached in an in vivo framework. An original model-based approach, expressed as a system identification problem, is proposed. The latter approach involves to study the practical identifiability of the photoreaction model and the estimation of its parameters. The main difficulties of this inverse problem are (i) the non linearity of the model structure and (ii) the lack of information in the experimental data (small data sets, small sampling rate, no measurement during the treatment phase (Laser ON), few degrees of freedom concerning the choice of the input signal and only one output variable for six state variables). The local practical identifiability test is based on the conditioning of the Fisher Information Matrix. The parameter estimation relies on a least-squares method applied to the output error. Despite the previously mentioned difficulties, the results obtained in practice from in vivo experiments have shown promising results.

\section{Perspectives}

Two main developments could enhance the accuracy of the estimates:

- designing new experiment protocols, i.e. seeking the optimal input signal $u_{L}$ and increasing significantly the sampling rate $f_{S}$ during the dark phases (laser OFF) of the test. A new research platform equipped with a laser diode is currently developed in order to both increase the signal-to-nose ratio and the degrees of freedom concerning the choice of the input signal;

- using global sensitivity approaches instead of the local approach used herein.

\section{ACKNOWLEDGMENTS}

We gratefully acknowledge L. Denis-Vidal (Université des Sciences et Technologie de Lille, France) for her scientific support in structural identifiability in this study.

\section{REFERENCES}

[1] J. G. Moser, Photodynamic Tumor Therapy: 2nd and 3rd Generation. Gordon \& Breach Science Publishers, 1998.

[2] M. P. Goldman, Photodynamic Therapy. W.B. Saunders Company, 2005

[3] D. C. Hamilton, D. G. Watts and D. M. Bates, Accounting for Intrinsic Nonlinearity in Nonlinear Regression Parameter Inference Regions, Ann. Statist, vol. 10, no. 2, pp. 386-393,1982.

[4] M. Niedre, M. S. Patterson, and B. C. Wilson, "Direct near-infrared luminescence detection of singlet oxygen generated by photodynamic therapy in cells in vitro and tissues in vivo, Photochemistry and Photobiology, vol. 75, no. 4, pp. 382-391, 2002.

[5] M. Niedre, C. Yu, M. Patterson, and B. Wilson, "Singlet oxygen luminescence as an in vivo photodynamic therapy dose metric: validation in normal mouse skin with topical amino-levulinic acid, British Journal of Cancer, vol. 92, pp. 298 - 304, 2005. 

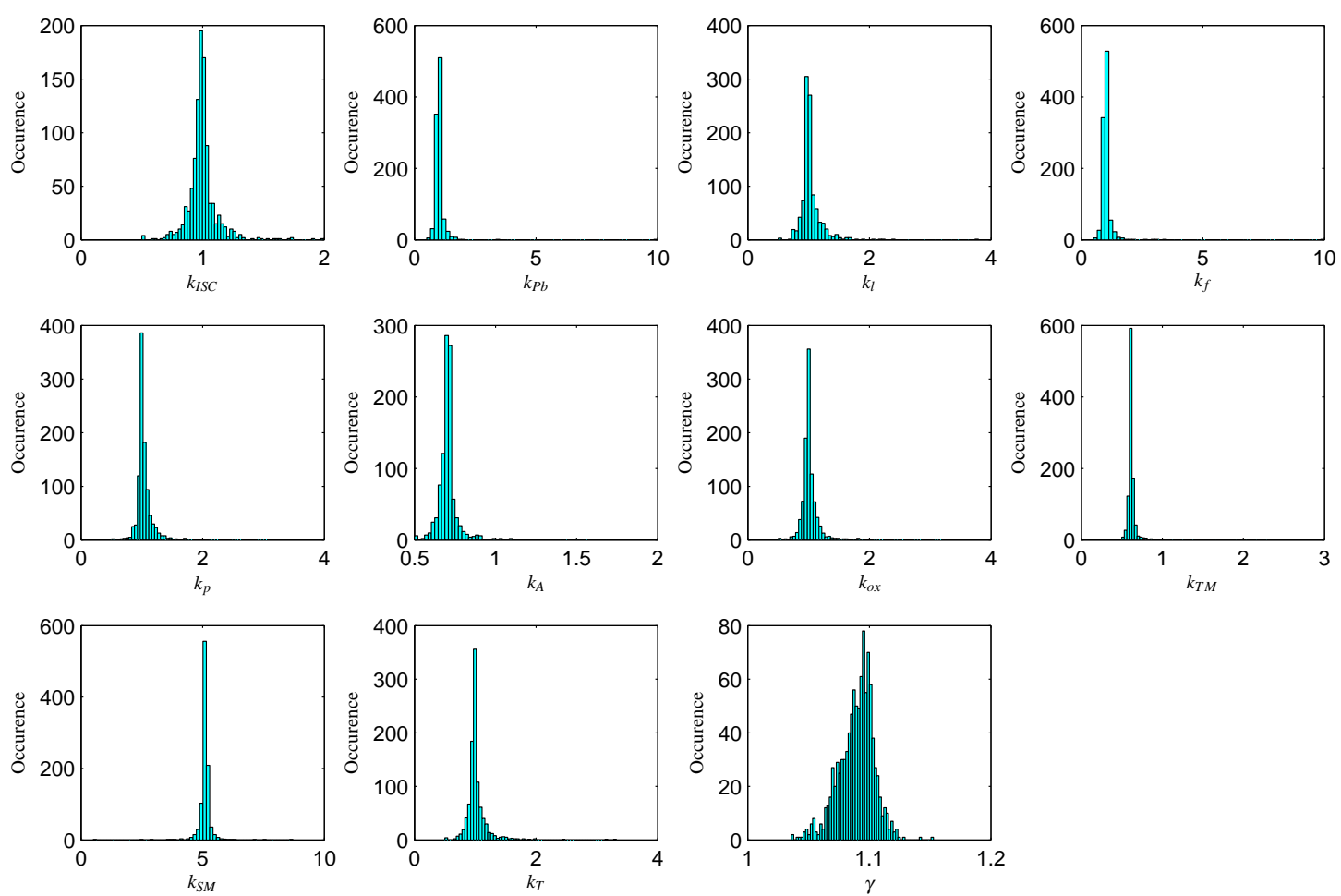

Fig. 5. Results of the Uncertainty Analysis using Monte Carlo method for the estimated parameters

[6] S. Marsili-Libelli, S. Guerrizio, and N. Checchi, "Confidence regions of estimated parameters for ecological systems, Ecological Modelling, vol. 165 , no. 20 , pp. 127-146, 2003.

[7] T. Bastogne, L. Tirand, S. Dobre, and M. Barberi-Heyob, "Modélisation systéme de la thérapie photodynamique, Revue e-STA Sciences et Technologies de l'Automatique, vol. 3, no. 2, 2006.

[8] T. Bastogne, M. Thomassin, and J. Masse, "Selection and identification of physical parameters from passive observation. application to a winding process, Control Engineering Practice, vol. 15, no. 9, pp. 1051-1061, 2007.

[9] R. Bonnett, B.D. Djelal, P.A. Hamilton,G. Martinez, F. Wierrani, "Photobleaching of 5,10,15,20-tetrakis(m-hydroxyphenyl)porphyrin (m-THPP) and the corresponding chlorin (m-THPC) and bacteriochlorin(m-THPBC). A comparative study", in Journal of Photochemistry and Photobiology, 1999, (53), pp. 136-143.

[10] A. M. Braun, E. Oliveros, "Applications of singlet oxygen reactions: mechanistic and kinetic investigations", Pure \& Appl. Chem., 1990, (62), pp. 1467-1476.

[11] M. C. DeRosa, R. J. Crutchley, "Photosensitized singlet oxygen and its applications", Coordination Chemistry Reviews, 2002, pp. 351-371.

[12] T. J. Dougherty, C. J. Gomer, B. W. Henderson, G. Jori, D. Kessel, M. Korbelik, J. Moan, Q. Peng, Photodynamic Therapy, J Natl Cancer Inst., vol. 90, pp. 889 - 905, 1998.

[13] T. Foster, R. S. Murant, R. G. Bryant, R. S. Knox, S. L. Gibson, and R. Hilf, "Oxygen consumption and diffusion effects in photodynamic therapy, Radiation Research, vol. 126, pp. 296-303, 1991.

[14] E. Walter and L. Pronzato, Identification of Parametric Models from experimental data. Springer-Verlag, Masson, 1997.

[15] E. Walter, Identifiability of Parametric Models,Pergamon, Oxford, 1987.

[16] S. Dobre, T. Bastogne, M. Barberi-Heyob and A. Richard, " Practical identifiability of photophysical parameters in photodynamic therapy, Engineering in Medicine and Biology Society, 2007, EMBC'2007, Lyon : France (2007).

[17] M. S. Grewal and K. Glover, "Identifiability of linear and nonlinear dynamical systems, IEEE Trans. on Automatic Control, vol. 21, no. 6, pp. 833-837, 1976.

[18] J. Masse, "Differentiation, analyze sensibilite and identification of the hybrid models decrits under Simulink, Revue de l'Electricite et de l'Electronique, no. 9, pp. 78-83, 2003.

[19] P. Vanrolleghem, M. Van Daele, and D. Dochain, "Practical identifiability of a biokinetic model of activated sludge respiration, Wat. Res., vol. 29 , no. 11 , pp. 2561-2570, 1995.

[20] D. Dochain and P. Vanrolleghem, Dynamical Modelling and Estimation in Wastewater Treatment Processes. IWA Publishing, 2001.

[21] D. De Pauw, Optimal Experimental Design for Calibration of Bioprocess Models: A Validated Software Toolbox, Faculty of Bioscience engineering. Ghent University, Belgium, 2005.

[22] S. Pinel, M. Barberi-Heyob, E. Cohen-Jonathan, J. L. Merlin, C. Delmas, F.Plenat, P. Chastagner, Erythropoietin-induced reduction of hypoxia before and during fractionated irradiation contributes to improvement of radioresponse in human glioma xenografts, Int. j. radiat. oncol. biol. phys.,2004, vol. 59, pp. 250-259.

[23] L. Tirand, N. Thomas, M. Dodeller, D. Dumas, C. Frochot, F. Guillemin and M. Barberi-Heyob (2007). Metabolic profile of a peptide-conjugated chlorintype photosensitizer targeting neuropilin-1: an in vivo and in vitro study. Drug Metab. Dispos. 35, 806813.

[24] S. Marsili-Libelli and E. Giusti, Water quality modelling for small river basins, Environmental Modelling \& Software 23 (2008), 451-463. 